\title{
SPECTRA OF BEAM INDUCED FIELDS IN CESR SUPERCONDUCTING CAVITIES*
}

\author{
V. Veshcherevich ${ }^{\dagger}$ and S. Belomestnykh \\ Laboratory of Nuclear Studies, Cornell University, Ithaca, NY 14853, USA
}

\begin{abstract}
Frequency spectra of the fields induced by beam in four superconducting accelerating cavities of CESR collider are measured and analyzed. Resonance frequencies and $Q$ values of cavity higher order modes are extracted from these measurements. Most of higher order modes have $Q$ values less than 2000. However, few of them have higher $Q$. The results are compared with computer calculations of higher order modes.
\end{abstract}

\section{INTRODUCTION}

The Cornell Electron Storage Ring (CESR) is a single ring symmetric electron-positron collider operating near the $Y(4 \mathrm{~S})$ resonance $(E \approx 5.3 \mathrm{GeV})$. The peak luminosity achieved in CESR is $1.3 \times 10^{33} \mathrm{~cm}^{-2} \mathrm{sec}^{-1}$. It operates with nine bunch trains in each beam (five $19 \mathrm{~mm}$ bunches spaced by $4.2 \mathrm{~m} / 14 \mathrm{nsec}$ in a train), with a total beam current of up to $780 \mathrm{~mA}$. The upgrade of CESR performance was achieved due to general machine upgrade, in particular due to upgrade of the CESR RF system.

Presently, the upgraded accelerating system of CESR [1] has four $500 \mathrm{MHz}$ single-cell superconducting niobium cavities in operation that replaced the old 7-cell copper cavities. The cavities are installed into CESR in pairs where they are spaced by $3 \mathrm{~m}$ ( 5 wave lengths).

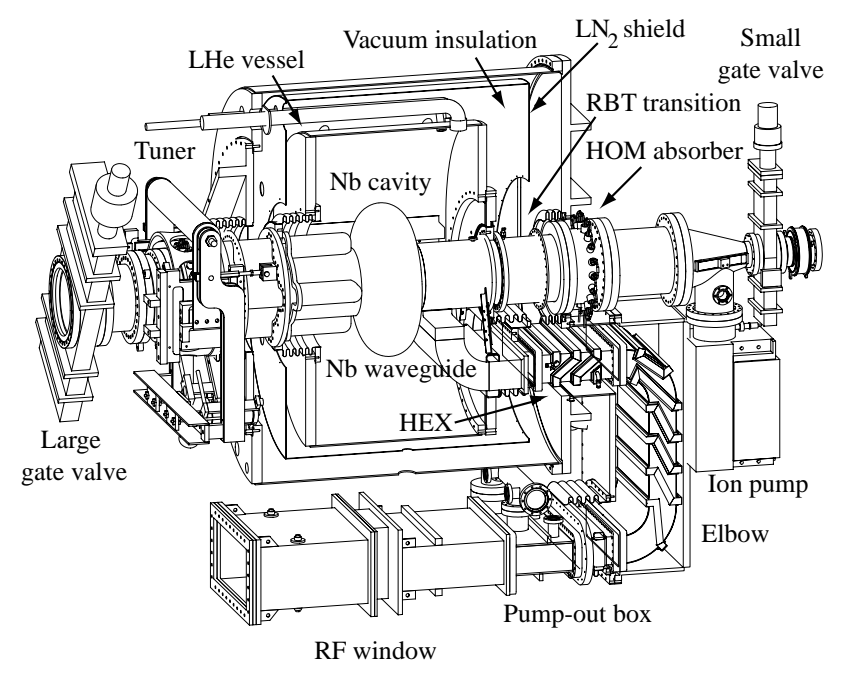

Figure 1: Layout of a CESR cryomodule.

\footnotetext{
* Work supported by the National Science Foundation.

$\dagger$ E-mail address:.vgv@1ns62.lns.cornell.edu.
}

Each cavity is mounted in an individual cryostat (see Figure 1). The cavity [2] is designed to have low impedances of higher order modes (HOMs). It is achieved due to bell shaped geometry and large diameter $(24 \mathrm{~cm})$ beam tubes that connect the cavity to ferrite RF absorbers located outside the cryostat. Higher order modes induced in the cavity by the beam can propagate in the beam tubes and be absorbed in ferrite loads at both sides of the cavity. One of the wide beam pipes is made fluted between the cavity and the ferrite absorber for ensuring propagation of lowest dipole modes to the absorber. At one cavity side the beam tube tapers to the regular CESR beam tube size behind the ferrite load. At the other cavity side the wide beam tube spreads to the adjacent cavity in the pair.

\section{MODE CALCULATIONS}

Since higher order modes can propagate in the wide beam tube between the cavities in a pair, two cavities in a pair are strongly coupled in terms of higher order modes and should be considered as a single two-cell cavity module in HOM calculations.

Calculations of higher order modes of the cavity module [3], [4], [5] were done using the CLANS computer code [6] taking into account actual lossy properties of the ferrite HOM loads. CLANS is a 2D code and therefore the module geometry was simplified for the calculations: a regular wide beam tube was considered instead of a fluted one. However, this simplification should have only a small effect on results

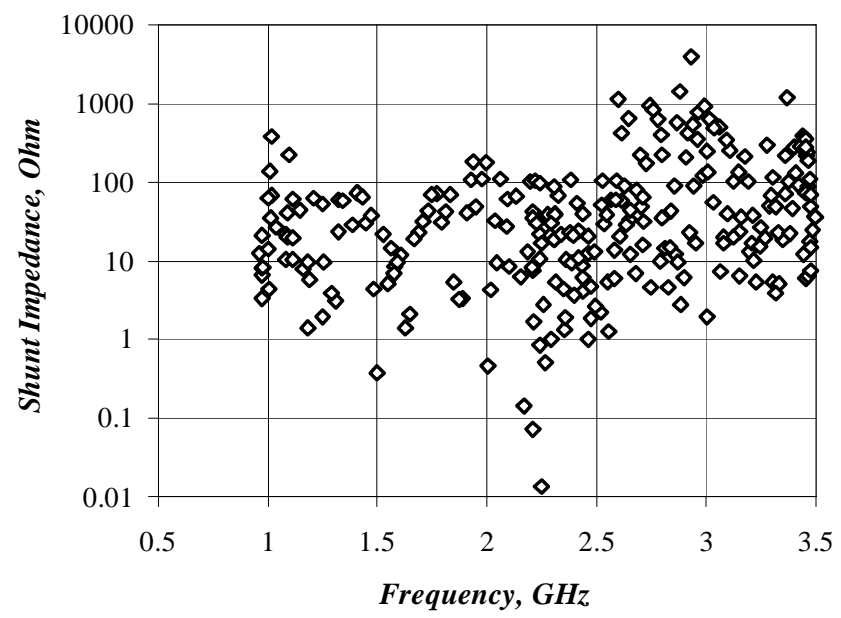

Figure 2: Calculated shunt impedances of monopole higher order modes of a two-cavity module. 


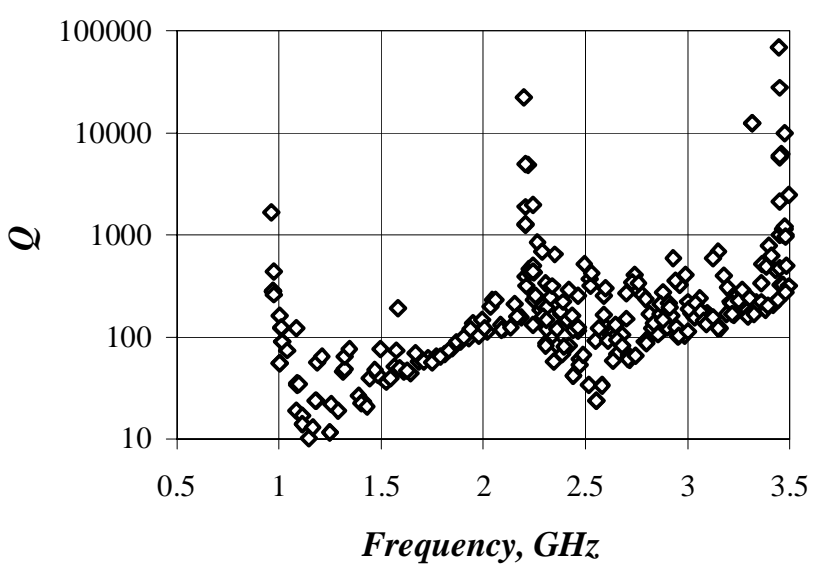

Figure 3: Calculated $Q$ values of monopole higher order modes of a two-cavity module.

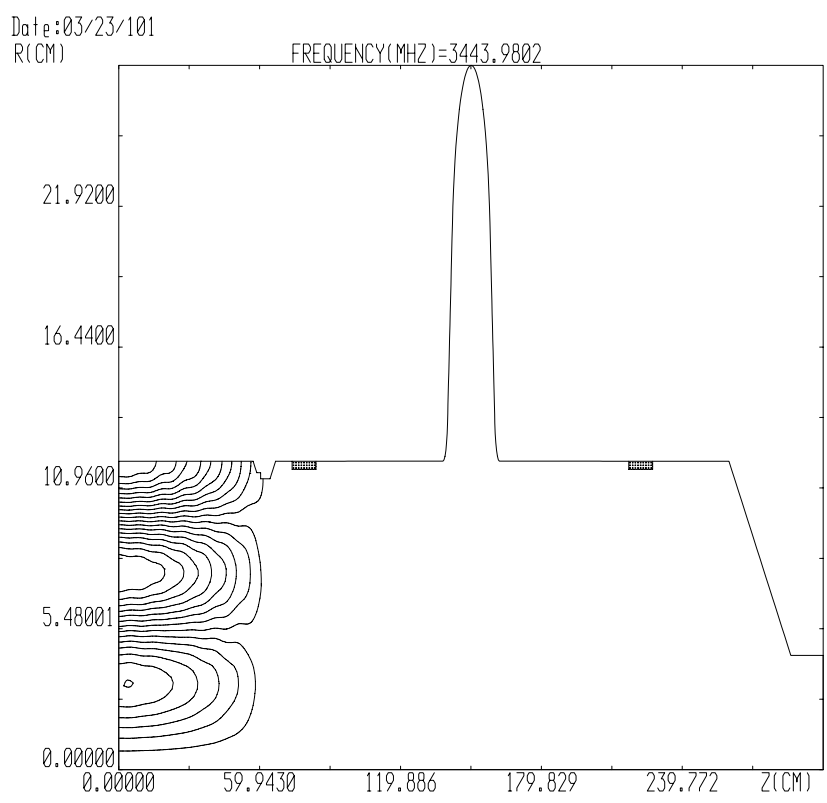

Figure 4: Field lines of a high $Q$ "trapped" mode. (A half of a two-cavity module is shown. Note that the scales on $z$ and $r$ axes are different).

for monopole modes (modes with no angular field variations) that are the most important for beam-cavity interaction. Figures 2 and 3 display calculated shunt impedances and $Q$ values of monopole HOMs for a cavity module. As it is seen in the Figures, most of higher order modes have low $Q, 10$ to 1000 and impedances lower than $100 \mathrm{Ohm}$. However, there are modes with higher $Q$. Those modes are so called trapped modes that have low fields is the regions of ferrite loads. It turned out their frequencies are close to cut-off frequencies of $T M_{01}, T M_{02}$ and $T M_{03}$ modes in the wide beam tube $(956 \mathrm{MHz}$, $2195 \mathrm{MHz}$ and $3441 \mathrm{MHz}$ respectively). Field lines of one of those modes are shown in Figure 4. Fortunately,

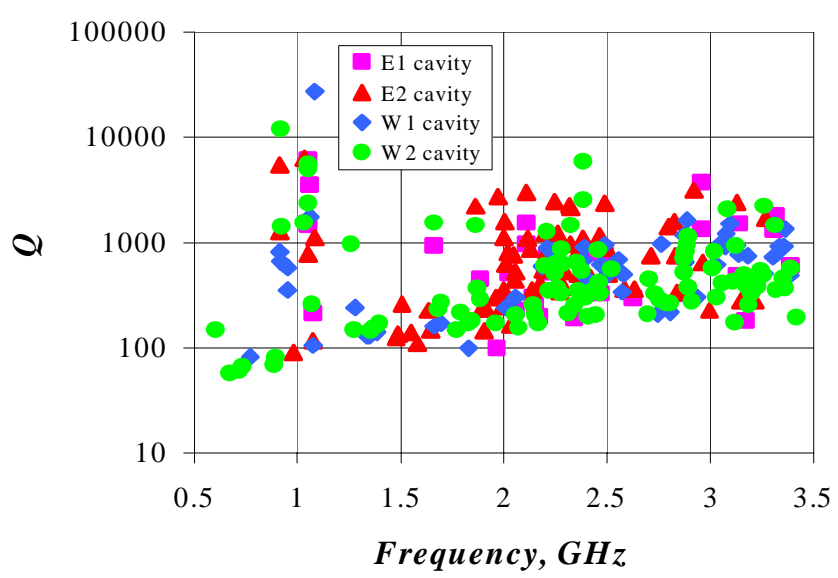

Figure 5: Measured HOM $Q$ values of four CESR cavities.

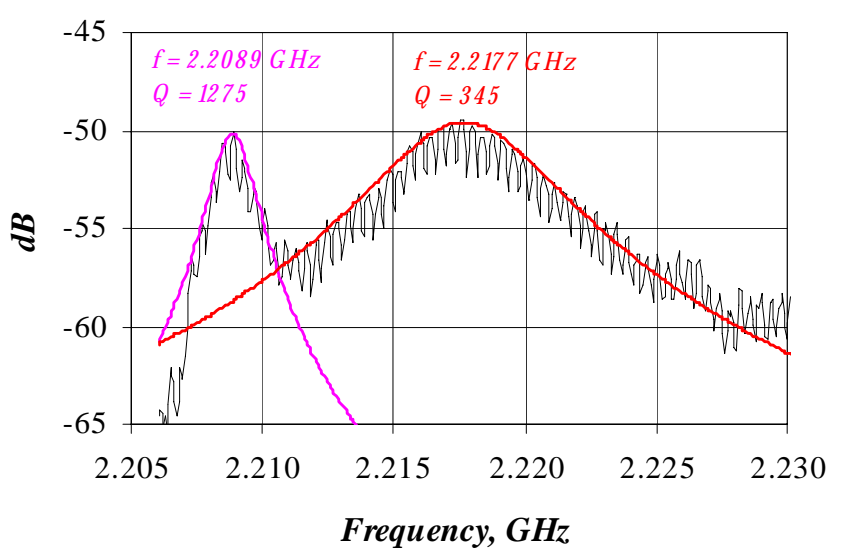

Figure 6: Two of higher order modes induced by the beam in a cavity.

those "trapped" modes have very low $R / Q$ values and-as it is seen in Figure 2-their shunt impedances are no higher than shunt impedances of low $Q$ modes.

\section{BEAM MEASUREMENTS}

In a single bunch mode operation, at a low beam current (4 to $4.5 \mathrm{~mA}$ ) frequency spectra of beam induced fields were measured by a spectrum analyzer for all four cavities installed in CESR. Antenna type field probes mounted in wide beam tubes were used for this purpose. Resonance peaks were searched in beam induced field spectra, approximation curves were fit for each peak and the resonance frequencies and $Q$ values of cavity modes were extracted. In those experiments the bunches were moving on the basic (undisturbed) CESR beam orbit - that means they passed the cavities close to the central axis. Therefore, the beam principally excited monopole modes in the cavities. The results are presented in Figure 5. Figure 6 shows an example of beam-induced signal where 
two higher order modes are displayed, with approximation curves and mode parameters.

\section{DISCUSSION}

There are some differences between calculated and measured HOM properties that can be seen from comparison of Figures 3 and 5.

Modes found in the cavities installed in the West part of CESR tunnel (especially in the cavity \#2-West) start at lower frequencies than the calculated monopole modes. Those low frequency modes $(f<950 \mathrm{MHz})$ are apparently dipole modes excited due to a small residual transverse offset of the beam. It was confirmed in experiments when we saw low frequency modes in other cavities too doing the measurements with an off-axis beam displacement. There are a certain number of dipole modes among other modes also. We need to do additional experiments and analyses to filter all dipole modes from cavity spectra.

Despite a certain amount of dipole modes among all measured modes the total number of modes found is less than it was predicted by computer calculations. One reason of that is a difficulty in finding low $Q$ modes $(Q<100)$. Their peaks are very wide, they overlap and actually form a general background. That partially explains why the average measured $Q$ values are generally higher (about a half of an order of magnitude) than the average values obtained by computer calculations-we could not see low $Q$ modes.

On the other hand, some high $Q$ modes predicted by calculations were not found also. Apparently, it was due to very low field level in the antenna probe area for those "trapped" modes. And even regular modes having a node of electrical field near the field probe could be overlooked.

Nevertheless, most of higher order modes found in the new CESR superconducting cavities have $Q$ values less than 2000. That is an evidence of satisfactory HOM damping.

\section{REFERENCES}

[1] S. Belomestnykh et al. "Running CESR at High Luminosity and Beam Current with Superconducting RF System". Proc. of the $7^{\text {th }}$ European Part. Accel. Conf. (Vienna, 2000), Vol. 3, pp. 2025-2027.

[2] H. Padamsee et al. "Design Challenges for High Current Storage Rings". Part. Accel., Vol. 40, pp. 17-42 (1992).

[3] B. Vakoc. "HOM Spectra and Dissipated Power Density Profiles for the SRF Cavity with a Ferrite HOM Load: Calculations with CLANS and Analysis". Cornell LNS Report SRF950511-11.

[4] R. Paryl. "High Order Mode Analysis for Two SRF Cavity Model. Monopole Modes". Cornell LNS Report SRF981118-15.

[5] C. Chen. "Calculations for Unfluted Two Cell Cavities". Cornell LNS Report SRF 000105-01.

[6] D. G. Myakishev and V. P. Yakovlev. "The new possibility of SUPERLANS Code for Evaluation of Axisymmetric Cavities". Proc. of the 1995 Part. Accel. Conf. (Dallas, TX, 1995), Vol. 4, pp. 23482350 . 